\title{
Anti-Müllerian Hormone (AMH) in Gynecological Endocrinology
}

\author{
Akmal El-Mazny* \\ Department of Obstetrics and Gynecology, Faculty of Medicine, Cairo University, Egypt
}

*Corresponding Author: Akmal El-Mazny, Department of Obstetrics and Gynecology, Faculty of Medicine, Cairo University, Egypt, Email: dr_akmalelmazny@yahoo.com

\begin{abstract}
Anti-Müllerian Hormone (AMH) is largely known as an indicator of ovarian reserve. This review discusses the different uses of AMH in gynecological endocrinology including guiding and monitoring of assisted reproduction treatment, the debate of AMH levels in polycystic ovary syndrome (PCOS), the role of $A M H$ measurements in premature ovarian failure (POF), as well as its value in pediatric endocrinology.
\end{abstract}

Keywords: Anti-Müllerian Hormone, AMH, Gynecological Endocrinology, Ovarian Reserve, Assisted Reproduction

\section{INTRODUCTION}

Anti-Müllerian hormone (AMH), also known as Mullerian inhibiting substance (MIS), is a dimeric protein member of the TGF- $\beta$ subfamily (1). AMH is produced by the granulosa cells of pre-antral and small antral follicles. Follicular growth is modulated by $\mathrm{AMH}$, which inhibits recruitment of follicles from the primordial pool by modifying the FSH sensitivity of those follicles. As a follicle matures, AMH production disappears allowing the follicle to complete the development process (2).

\section{DISCUSSION}

\subsection{Ovarian Reserve}

Ovarian reserve represents the remaining population of primordial and resting follicles; and is generally defined as the quantity and quality of the follicles present in the ovary (3). Although chronological age is the major determinant of ovarian reserve, there is considerable individual variability in the rate of ovarian ageing (4). Therefore, accurate evaluation of ovarian reserve may allow individualized patient management.

Traditionally, other than age, basal (day 3) serum follicle stimulating hormone (FSH) level and antral follicle count (AFC) assessed by trans-vaginal sonography are often used as tests of ovarian reserve. FSH is not considered highly accurate due to inter-cycle variations (3). AFC correlates with the number of oocytes (5); and is considered to be one of the best tests of ovarian reserve, and much better than FSH or inhibin B assays (6).

\subsection{Assisted Reproduction}

Assisted reproduction (IVF/ICSI) treatment of infertility results in reasonably high pregnancy rates; however, the variability in patient response dictates the need for personalized diagnostic and therapeutic approach to optimize efficacy and safety outcomes (7).The ovarian response to gonadotrophin stimulation depends primarily on the ovarian reserve; and is a major determinant of the success of assisted reproduction (8).

The clinical significance of AMH determination was first proven in assisted reproduction, as AMH levels reflect the ovarian reserve potential with high accuracy (9-12). There is also a strong correlation basal AMH level and the number of retrieved oocytes $(10,12)$. The fact that $\mathrm{AMH}$ is secreted without dependence on other hormones, particularly the gonadotropins; and at a constant level, independent of cycle day, makes AMH very attractive as a direct measure of ovarian reserve (13).

Compared to other ovarian reserve biomarkers, Themmen(14) found AMH to be a better marker to predict the ovarian response to gonadotropin stimulation than age, day $3 \mathrm{FSH}$, estradiol, and inhibin B. Gleicher et al. (15)also concluded that women with normal FSH and abnormal AMH 
will have reduced oocyte yield, and women with normal FSH and normal AMH will have the best oocyte yield; showing again that $\mathrm{AMH}$ is a better marker than FSH.

Compared to AFC, Broer et al. (16) concluded that both AMH and AFC have the same accuracy level in predicting ovarian response and outcome after IVF. This was confirmed by another study that showed that small AFC (2-6 $\mathrm{mm})$ and $\mathrm{AMH}$ are equally accurate predictors of ovarian response to controlled ovarian hyperstimulation (17). In addition to being a good marker for the quantity of follicles, AMH is also suggestive of the quality of the remaining oocytes (18).

Furthermore, AMH has the ability to predict both poor response and excessive response to gonadotropins. Compared to AFC, AMH concentrations could reliably and equally predict poor response to ovarian stimulation in IVF cycles (19). On the opposite end of the spectrum, it was reported that AMH levels could recognize those women prone to ovarian hyper stimulation syndrome (OHSS) during ovulation induction with gonadotropins (20).

\subsection{Polycystic Ovary Syndrome (PCOS)}

Since AMH levels reflect the number of developing follicles, their measurement may be used as a marker of ovarian follicle impairment in PCOS. It has been shown that circulating AMH levels are higher in PCOS patients, and that AMH levels correlate with the severity of the syndrome (21). However, AMH has not proven as a diagnostic marker of PCOS (22).

\subsection{Premature Ovarian Failure (POF)}

AMH could be a marker for women at risk for POF. In cancer survivorsafter chemotherapy and/or radiotherapy, AMH levels correlate with the extent of gonadal damage (23). Early diagnosis of POF can prevent expensive ways of fertility treatment. AMH is a slightly better marker of POF than FSH; having more sensitivity (24).

\subsection{Turner Syndrome}

AMH levels can serve as a useful marker of the follicle pool in patients with Turner syndrome. $\mathrm{AMH}$ levels in these patients are related to karyotype, pubertal development and growth hormone treatment (25). A low AMH level can predict failure to enter puberty in young patients and imminent POF (26).

\subsection{Precocious Puberty}

AMH levels in girls with precocious puberty are usually in the normal range. However, AMH levels decrease at the early stages of GnRH agonist treatment, but return to pretreatment levels after discontinuation of the treatment, indicating $\mathrm{GnRH}$ treatment does not affect fertility (27).

\subsection{Female Virilization}

$\mathrm{AMH}$ levels can indicate if the virilization originates from testicular tissue or of a granulosa cell tumor -- AMH levels in the male reference range, or from adrenal androgens, as in the case of congenital adrenal hyperplasia -$\mathrm{AMH}$ levels in the female reference range (28).

\section{CONCLUSION}

AMH measurement is of proven value for estimating ovarian reserve and guiding and monitoring of assisted reproduction treatment. The use of $\mathrm{AMH}$ as a diagnostic marker in PCOS is still controversial. AMH measurements to predict POFafter chemotherapy and/or radiotherapy of cancer are promising. It is also a useful tool in the field of pediatric endocrinology.

\section{REFERENCES}

[1] La Marca A, Volpe A. Anti-Mullerian hormone $(\mathrm{AMH})$ in female reproduction: is measurement of circulating AMH a useful tool? ClinEndocrinol (Oxf). 2006; 64: 603-10.

[2] Visser JA, de Jong FH, Laven JS, Themmen AP. Anti-Mullerian hormone: a new marker for ovarian function. Reproduction. 2006; 131:1-9.

[3] Broekmans FJ, Kwee J, Hendriks DJ, et al. A systematic review of tests predicting ovarian reserve and IVF outcome. Hum Reprod Update. 2006; 12: 685-718.

[4] teVelde ER, Pearson PL. The variability of female reproductive ageing. Hum Reprod Update. 2002; 8: 141-54.

[5] Frattarelli JL, Levi AJ, Miller BT, et al. A prospective assessment of the predictive value of basal antral follicles in in vitro fertilization cycles. FertilSteril. 2003; 80: 350-5.

[6] Bancsi LF, Broekmans FJ, Eijkemans MJ, et al. Predictors of poor ovarian response in in vitro fertilization: a prospective study comparing basal markers of ovarian reserve. FertilSteril. 2002; 77: 328-36.

[7] Devroey P, Fauser BC, Diedrich K; Evian Annual Reproduction (EVAR) Workshop Group 2008. Approaches to improve the diagnosis and management of infertility. Hum Reprod Update. 2009; 15: 391-408.

[8] Shanbhag S, Aucott L, Bhattacharya S, et al. Interventions for 'poor responders' to controlled ovarian hyperstimulation $(\mathrm{COH})$ in in-vitro fertilisation (IVF). Cochrane Database Syst Rev. 2007:CD004379. 
[9] Sowers MR, Eyvazzadeh AD, McConnell D, et al. Anti-Mullerian hormone and inhibin B in the definition of ovarian aging and the menopause transition. J ClinEndocrinolMetab. 2008; 93: 3478-83.

[10] La Marca A, Broekmans FJ, Volpe A, et al. ESHRE Special Interest Group for Reproductive Endocrinology-AMH Round Table. Anti-Mullerian hormone (AMH): what do we still need to know? Hum Reprod. 2009; 24: 2264-75.

[11] Van Disseldorp J, Lambalk CB, Kwee J, et al. Comparison of inter-and intra-cycle variability of anti-Mullerian hormone, antral follicle counts. Hum Reprod. 2009; 25: 221-7.

[12] La Marca A, Sighinolfi G, Radi D, et al. AntiMullerian hormone (AMH) as a predictive marker in assisted reproductive technology (ART). Hum Reprod Update. 2010; 16: 113-30.

[13] Karkanaki A, Vosnakis C, Panidis D. The clinical significance of anti-Müllerian hormone evaluation in gynecological endocrinology. Hormones. 2011; 10: 95-103.

[14] Themmen AP. Anti-Mullerian hormone: its role in follicular growth initiation and survival and as an ovarian reserve marker. J Natl Cancer InstMonogr. 2005; 34: 18-21.

[15] Gleicher N, Weghofer A, Barad D. Discordances between follicle stimulating hormone $(\mathrm{FSH})$ and anti-Mullerian hormone $(\mathrm{AMH})$ in female infertility. ReprodBiolEndocrinol. 2010; 8: 64.

[16] Broer SL, Mol BW, Hendriks D, et al. The role of anti-Mullerian hormone in prediction of outcome after IVF: comparison with the antral follicle count. FertilSteril. 2008; 22: 193-201.

[17] Aflatoonian A, Oskouian H, Ahmadi S, et al. Prediction of high ovarian response to controlled ovarian hyperstimulation: antiMüllerian hormone versus small antral follicle count (2-6 mm). J Assist Reprod Genet. 2009; 26: $319-25$.

[18] Ebner T, Sommergruber M, Moser M, et al. Basal level of anti-Mullerian hormone is associated with oocyte quality in stimulated cycles. HumReprod. 2006; 21: 2022-6.

[19] Broer S, Mol BW, Dolleman M, et al. The role of anti-Müllerian hormone assessment in assisted reproductive technology outcome. CurrOpinObstet Gynecol. 2010; 22: 193-201.

[20] Nakhuda GS, Chu MC, Wang J, et al. Elevated serum MIS levels may be a marker for ovarian hyperstimulation syndrome in normal women undergoing IVF. FertilSteril. 2006; 85: 1541-3.

[21] Begawy AF, El-Mazny AN, Abou-Salem NA, et al. Anti-Müllerian hormone in polycystic ovary syndrome and normo-ovulatory women: Correlation with clinical, hormonal and ultrasonographic parameters. Middle East Fertility Society Journal. 2010; 15: 253-8.

[22] Dewailly D, Gronier H, Poncelot E, et al. Diagnosis of polycystic ovary syndrome (PCOS): revisiting the treshold values of follicle count on ultrasound and of the serum AMH level for the definition of polycystic ovaries. Hum.Reprod. 2011; 26: 3123-29.

[23] Visser JA,Schipper I,Laven JS,et al. AntiMüllerianhormone: an ovarian reserve marker in primary ovarian insufficiency.Nat Rev Endocrinol. 2012; 8:331-43.

[24] Alipour F, Rasekhjahromi A, Maalhagh M, et al. Comparison of Specificity and Sensitivity of $\mathrm{AMH}$ and $\mathrm{FSH}$ in Diagnosis of Premature Ovarian Failure. Dis Markers. 2015; 2015: 585604.

[25] Visser JA, Hokken-Koelenga AC, Zandwijken GP, et al.Anti-Müllerian hormone levels in girls and adolescents with Turner syndrome are related to karyotype, pubertal development and growth hormone treatment. Hum Reprod. 2013; 28: 1899-907.

[26] Lunding SA, Aksglaede L, Anderson PA,et al.AMH as Predictor of Premature Ovarian Insufficiency: A Longitudinal Study of 120 Turner Syndrome Patients. J ClinEndocrinolMetab. 2015; 100: E 1030-8.

[27] Hyo KH, Kim HR, Rhie YJ, et al. Serum AntiMüllerian Hormone Levels in Precocious Puberty Girls according to Stage of GnRH Agonist Treatment. J Korean Med Sci. 2017; 3: 475-79.

[28] Misra M, MacLaughlin DT, Donahoe PK. The role of Müllerian inhibiting substance in the evaluation of phenotypic female patients with mild degree of virilization. J ClinEndocrinolMetab. 2003; 88: 787-92.

Citation: Akmal El-Mazny. Anti-Müllerian Hormone (AMH) in Gynecological Endocrinology. ARC Journal of Gynecology and Obstetrics 2017; 2(4):17-19. DOI: dx.doi.org/10.20431/2455-9792.0204004.

Copyright: (C) 2017 Authors. This is an open-access article distributed under the terms of the Creative Commons Attribution License, which permits unrestricted use, distribution, and reproduction in any medium, provided the original author and source are credited. 